\title{
Optimizing the relativistic energy density functional with nuclear ground state and collective excitation properties
}

\author{
E. Yüksel* \\ Department of Physics, Faculty of Science, University of Zagreb, Bijenička c. 32, 10000 Zagreb, Croatia and \\ Department of Physics, Yildiz Technical University, 34220 Esenler, Istanbul, Turkey \\ T. Marketin ${ }^{\dagger}$ and N.Paar \\ Department of Physics, Faculty of Science, University of Zagreb, Bijenička c. 32, 10000 Zagreb, Croatia
}

(Dated: January 18, 2019)

\begin{abstract}
We introduce a new relativistic energy density functional constrained by the ground state properties of atomic nuclei along with the isoscalar giant monopole resonance energy and dipole polarizability in ${ }^{208} \mathrm{~Pb}$. A unified framework of the relativistic Hartree-Bogoliubov model and random phase approximation based on the relativistic density-dependent point coupling interaction is established in order to determine the DD-PCX parameterization by $\chi^{2}$ minimization. This procedure is supplemented with the co-variance analysis in order to estimate statistical uncertainties in the model parameters and observables. The effective interaction DD-PCX accurately describes the nuclear ground state properties including the neutron-skin thickness, as well as the isoscalar giant monopole resonance excitation energies and dipole polarizabilities. The implementation of the experimental data on nuclear excitations allows constraining the symmetry energy close to the saturation density, and the incompressibility of nuclear matter by using genuine observables on finite nuclei in the $\chi^{2}$ minimization protocol, rather than using pseudo-observables on the nuclear matter, or by relying on the ground state properties only, as it has been customary in the previous studies.
\end{abstract}

PACS numbers: 21.60.Ev, 21.60.Jz, 21.65.Ef,24.30.Cz,24.30.Gd,25.20.-x

Solving the quantum many-body problem of strongly interacting nucleons represents one of the fundamental challenges not only for understanding the phenomena of nuclear structure and dynamics, but also for various applications of astrophysical relevance, e.g., modeling the stellar evolution, supernova explosion, the properties of compact stars, the synthesis of chemical elements in the universe, etc. Among a variety of theoretical frameworks to address this problem, the nuclear energy density functional (EDF) represents a unified approach to study quantitatively static and dynamic properties of finite nuclei along the nuclide map $[1,2]$ as well as the equation of state of nuclear matter [3]. A considerable progress has been achieved in constructing and optimizing the phenomenological EDFs, both in non-relativistic [4-6] and relativistic [1, 7-9, 11] frameworks. Recently, the construction of the EDFs was also inspired by $a b i n i$ tio calculations [12] and effective field theories [13]. As pointed out in Ref. [14], strengths of the tensor forces guided by $a b$ initio relativistic Brueckner-Hartree-Fock calculations can also be used as a guide for the future $a b$ initio derivations of the EDFs. At present, only the phenomenological EDFs provide a level of accuracy required to quantitatively describe the nuclear properties across the whole nuclide map.

So far, the EDFs have mainly been parametrized with the experimental data on the ground state properties of nuclei. These observables alone are often not enough to constrain the effective interaction completely, especially its isovector channel, thus the protocols to determine the EDF's parameters often included constraints on the pseudo-observables on the nuclear matter properties. The neutron skin thickness $r_{n p}$, isovector dipole excitations in nuclei and neutron star mass and radii represent some of the possible observables that could be used to probe the isovector channel of the EDFs [3, 15, 16]. However, the data on $r_{n p}$ are often model dependent, and the most recent data from parity violating electron scattering experiment (PREX) on ${ }^{208} \mathrm{~Pb}$ [17] have large uncertainties, while the neutron star mass-radius data are still rather limited. An alternative observable to improve the performance of the EDF's isovector sector is the dipole polarizability $\left(\alpha_{D}\right)$, which is proportional to the inversely energy-weighted sum of the isovector dipole excitation in a nucleus. Recently, it has been represented as an observable strongly correlated with the symmetry energy parameters of the nuclear equation of state $[18,19]$. The dipole polarizability was also measured in several nuclei, including ${ }^{48} \mathrm{Ca},{ }^{68} \mathrm{Ni},{ }^{120} \mathrm{Sn}$ and ${ }^{208} \mathrm{~Pb}$ [20-23]. So far, mainly due to computational difficulties, the dipole polarizability was not employed to constrain the EDFs directly; instead, already established EDFs were tested for their performance in reproducing the experimental data on $\alpha_{D}$ [3]. On the other side, the isoscalar giant monopole resonance (ISGMR) energy in nuclei also represents an important observable to probe the isoscalar channel of the EDFs and incompressibility of nuclear matter $K_{0}$ [24]. To date, only in Ref. [11] the ISGMR was used directly in the optimization of an EDF (relativistic FSUGold2 interaction) along with the properties of finite nuclei and neutron stars.

The purpose of this work is to establish the first unified 
framework based on the EDF, that allows to constrain an effective interaction not only by the experimental data on nuclear ground state properties, but also by a direct implementation of the measured properties of collective nuclear phenomena: the ISGMR excitation energy and the dipole polarizability. In this way, the properties of nuclei and the nuclear equation of state; incompressibility of nuclear matter and the symmetry energy around the saturation density will be constrained directly by the experimental data on finite nuclei.

In the framework of the relativistic nuclear energy density functional, the nuclear ground-state density and energy are determined by the self-consistent solution of relativistic single-nucleon Kohn-Sham equations [25, 26 . In the present study these equations are implemented from an interaction Lagrangian density with four fermion contact interaction terms including the isoscalarscalar, isoscalar-vector, isovector-vector and isospacespace channels (for more details see Refs. [7, 27]),

$$
\begin{aligned}
\mathcal{L} & =\bar{\psi}(i \gamma \cdot \partial-m) \psi \\
& -\frac{1}{2} \alpha_{S}(\rho)(\bar{\psi} \psi)(\bar{\psi} \psi)-\frac{1}{2} \alpha_{V}(\rho)\left(\bar{\psi} \gamma^{\mu} \psi\right)\left(\bar{\psi} \gamma_{\mu} \psi\right) \\
& -\frac{1}{2} \alpha_{T V}(\rho)\left(\bar{\psi} \vec{\tau} \gamma^{\mu} \psi\right)\left(\bar{\psi} \vec{\tau} \gamma_{\mu} \psi\right) \\
& -\frac{1}{2} \delta_{S}\left(\partial_{\nu} \bar{\psi} \psi\right)\left(\partial^{\nu} \bar{\psi} \psi\right)-e \bar{\psi} \gamma \cdot A \frac{1-\tau_{3}}{2} \psi .
\end{aligned}
$$

In addition to the free-nucleon terms, the effective Lagrangian includes point coupling interaction terms, coupling of protons to the electromagnetic field, and the derivative term accounting for the leading effects of finiterange interactions necessary for a quantitative description of nuclear density distribution and radii. Starting from the microscopic density dependence of the scalar and vector self-energies, the following functional form of the couplings is employed [7, 28],

$$
\alpha_{i}(\rho)=a_{i}+\left(b_{i}+c_{i} x\right) e^{-d_{i} x}, \quad(i=S, V, T V),
$$

where $x=\rho / \rho_{0}$, and $\rho_{0}$ represents the nucleon density in symmetric nuclear matter at saturation point. The point-coupling model includes 10 parameters $\left(a_{S}, b_{S}, c_{S}\right.$, $d_{S}, a_{V}, b_{V}, d_{V} b_{T V}, d_{T V}$ and $\left.\delta_{S}\right)$. For the description of open-shell nuclei, the relativistic Hartree-Bogoliubov (RHB) model [27] is used and the pairing field is formulated using separable pairing force, which also includes two parameters for the pairing strength $\left(G_{p}\right.$ and $\left.G_{n}\right)$ [29]. More details and various implementations of the RHB model can be found in Refs. [30, 31]. In the small amplitude limit, the collective excitations are described by the relativistic (quasiparticle) random phase approximation (Q)RPA [2]. In the present study, a unified computational framework of the RHB model [27] and selfconsistent relativistic (Q)RPA [2] is established to constrain the 12 model parameters by minimizing the $\chi^{2}$ objective function [32]. In order to constrain the model parameters, the binding energies (34 nuclei), charge radii (26 nuclei) and mean pairing gaps (15 nuclei) of the selected open-shell nuclei are used along with the two observables on collective excitations: the constrained ISGMR energy $\left(E_{I S G M R}=\sqrt{m_{1} / m_{-1}}\right)$ and dipole polarizability for ${ }^{208} \mathrm{~Pb}$ (see the supplementary material for details on the data set). Here, $m_{1}$ and $m_{-1}$ represent the energy-weighted moment and inverse energyweighted moment of the strength distribution [2], respectively. The mean gap values for protons and neutrons are calculated using the five-point formula [33]. The adopted errors for the binding energies, charge radii and pairing gaps are taken as $1.0 \mathrm{MeV}, 0.02 \mathrm{fm}$ and $0.05 \mathrm{MeV}$, respectively. Recently, the dipole polarizability was measured in ${ }^{208} \mathrm{~Pb}$ using polarized proton inelastic scattering at extreme forward angles [20]. After the subtraction of the quasi-deuteron effect from the experimental data, the dipole polarizability was obtained as $19.6 \pm 0.6 \mathrm{fm}^{3}$ in ${ }^{208} \mathrm{~Pb}$ [18]. Several experimental studies were also performed to explore the ISGMR in ${ }^{208} \mathrm{~Pb}$ [34-38]. Although the uncertainties for the measured ISGMR energies are small, there are differences in the excitation energies from different studies. Recently, the ISGMR energies were measured for ${ }^{204,206,208} \mathrm{~Pb}$ using inelastic $\alpha$-scattering at extremely forward angles, and the constrained ISGMR energy was found as $13.5 \pm 0.1$ in ${ }^{208} \mathrm{~Pb}$ [34], whereas this energy was previously obtained as $14.18 \pm 0.11 \mathrm{MeV}$ in the Texas A\&M experiment [35]. In optimizing the EDF, the constrained ISGMR energy is taken as $13.5 \mathrm{MeV}$ [34] and due to experimental uncertainties we adopted slightly large error $(1.0 \%)$ in the fitting protocol. The dipole polarizability is also taken as $19.6 \mathrm{fm}^{3}$ [18] and the adopted error used is $0.5 \%$. As mentioned above, the implementation of the collective nuclear excitations in the fitting protocol is crucial in constraining both the isoscalar and isovector channels of the EDF's [3, 24].

Using the observables introduced above, the $\chi^{2}$ minimization for the relativistic point coupling interaction is performed. This procedure is supplemented with the co-variance analysis that allows to determine statistical uncertainties of the model parameters and other quantities, as well as relevant correlations between various properties [19, 32]. Accordingly, the curvature matrix is determined at the $\chi^{2}$ minimum, $\mathcal{M} \equiv \partial_{p_{i}} \partial_{p_{j}} \chi^{2}$, where $p_{i}$ and $p_{j}$ denote the interaction parameters $(i, j=1, . ., 12)$. Then, it is used to estimate the uncertainties of the model parameters, using $\sigma\left(p_{i}\right) \equiv \sqrt{\left(\mathcal{M}^{-1}\right)_{i i}}$ relation [32]. The covariance between the two observables $(A, B)$ is defined as [32]

$$
\operatorname{cov}(A, B)=\operatorname{cov}(B, A)=\sum_{i, j=1}^{N}\left(\frac{\partial A}{\partial p_{i}}\right) \mathcal{M}_{i j}^{-1}\left(\frac{\partial B}{\partial p_{j}}\right),
$$

where the derivatives of the observables and the inverse of the curvature matrix are calculated at the $\chi^{2}$ minimum. The statistical uncertainty of any quantity of interest $A$ 
is calculated using $\sigma(A)=\sqrt{\operatorname{cov}(A, A)}$.

The resulting DD-PCX parameterization with the respective statistical uncertainties are given in Table I. The statistical uncertainties of the parameters are found to be small, indicating that the parameters of the interaction are well constrained.

TABLE I. Parameters of the DD-PCX interaction with the corresponding statistical uncertainties. The value of the nucleon mass is $939.0 \mathrm{MeV}$ and the saturation density is set to $0.152 \mathrm{fm}^{-3}$.

\begin{tabular}{lcc}
\hline parameters & DD-PCX & $\sigma$ \\
\hline$a_{s}\left(\mathrm{fm}^{2}\right)$ & -10.979243836 & 0.010808546 \\
$b_{s}\left(\mathrm{fm}^{2}\right)$ & -9.038250910 & 0.023987420 \\
$c_{s}\left(\mathrm{fm}^{2}\right)$ & -5.313008820 & 0.047152813 \\
$d_{s}$ & 1.379087070 & 0.003900800 \\
$a_{v}\left(\mathrm{fm}^{2}\right)$ & 6.430144908 & 0.024888709 \\
$b_{v}\left(\mathrm{fm}^{2}\right)$ & 8.870626019 & 0.019549460 \\
$d_{v}$ & 0.655310525 & 0.003073028 \\
$b_{t v}\left(\mathrm{fm}^{2}\right)$ & 2.963206854 & 0.092150525 \\
$d_{t v}$ & 1.309801417 & 0.053360277 \\
$\delta_{s}\left(\mathrm{fm}^{4}\right)$ & -0.878850922 & 0.004512226 \\
\hline$G_{n}\left(\mathrm{MeV} \cdot \mathrm{fm}^{3}\right)$ & -800.663126037 & 6.279054350 \\
$G_{p}\left(\mathrm{MeV} \cdot \mathrm{fm}^{3}\right)$ & -773.776776597 & 4.003044910 \\
\hline
\end{tabular}

Table II shows the nuclear matter properties at the saturation for density-dependent point coupling interactions, DD-PCX (with uncertainties) and DD-PC1 [7], density dependent meson-exchange interaction DD-ME2 [8], and non-linear point coupling interaction PC-PK1 [9]. These include the energy per nucleon $E / A$, the Dirac effective nucleon mass $m_{D}^{*}[7]$, the nuclear matter compression modulus $K_{0}$, the symmetry energy at saturation density $J$ and the slope of the symmetry energy at saturation $L[3]$. Compared to the DD-PCX, the DD-PC1, DD-ME2, and PC-PK1 interactions were established using different protocols. The DD-ME2 parameterization is based on the density-dependent mesonexchange interaction, constrained using the ground state properties of spherical nuclei [8], whereas the DD-PC1 interaction is based on the point-coupling model, and optimized using the binding energies of deformed nuclei [7]. In addition, selected nuclear matter properties were fixed in both interactions, and no data on excitations have been used in the $\chi^{2}$ minimization. In Ref. [9] the non-linear point coupling interaction PC-PK1 has been constrained by fitting to observables of 60 selected spherical nuclei, including the binding energies, charge radii, and empirical pairing gaps, and no constraints on the nuclear matter properties have been used in the fitting protocol. Due to the implementation of the nuclear excitations for ${ }^{208} \mathrm{~Pb}$ in constraining the DD-PCX interaction, we find that the incompressibility $\left(K_{0}\right)$ and symmetry energy parameters $(J$ and $L)$ at saturation density are lower than for the DD-PC1, DD-ME2, and PC-PK1 effective interactions. The respective uncertainties are also found to be small, indicating that within the fitting protocol employed both the isoscalar and isovector channels of the interaction are tightly constrained. The DD-PCX interaction values for $J$ and $L$ are in agreement with the suggested values from previous studies [3]. The compression modulus $K_{0}$ is also found at around $213 \mathrm{MeV}$, which is lower in comparison to other relativistic interactions $[7,8]$. We realize that small $K_{0}$ is a direct consequence of the more recent experimental data $\left(E_{I S G M R}=13.5 \pm 0.1 \mathrm{MeV}\right)[34]$ used in the fitting proto$\mathrm{col}$, and the implementation of the data from Texas A\&M experiment $\left(E_{I S G M R}=14.18 \pm 0.11 \mathrm{MeV}\right)$ [35] would lead toward the higher value of $K_{0}$. Clearly, resolving the ambiguities between different experimental studies on the ISGMR data for ${ }^{208} \mathrm{~Pb}$ is essential for constraining the nuclear matter incompressibility.

TABLE II. The saturation properties of nuclear matter for the DD-PCX, DD-PC1 [7], DD-ME2 [8], and PC-PK1 [9] interactions.

\begin{tabular}{lcccc}
\hline & DD-PCX & DD-PC1 & DD-ME2 & PC-PK1 \\
\hline E/A $(\mathrm{MeV})$ & $-16.026 \pm 0.018$ & -16.06 & -16.14 & -16.12 \\
$m_{D}^{*} / m$ & $0.5598 \pm 0.0008$ & 0.580 & 0.572 & 0.590 \\
$K_{0}(\mathrm{MeV})$ & $213.03 \pm 3.54$ & 230.0 & 250.89 & 238.0 \\
$J(\mathrm{MeV})$ & $31.12 \pm 0.32$ & 33.0 & 32.30 & 35.60 \\
$L(\mathrm{MeV})$ & $46.32 \pm 1.68$ & 70.0 & 51.26 & 113.0 \\
\hline
\end{tabular}

In the following, the performance of the DD-PCX interaction in description of various nuclear ground state and excitation properties is presented. Figure 1 shows the differences between the experimental [39] and calculated binding energies (upper panel) and the charge radii for $\mathrm{Ca}, \mathrm{Ni}, \mathrm{Sn}$ and $\mathrm{Pb}$ isotopes (lower panel) for the $\mathrm{DD}$ PCX interaction. For comparison, the results are also shown for the density dependent point coupling interaction DD-PC1 [7], density dependent meson-exchange interaction DD-ME2 [8], and non-linear point coupling interaction PC-PK1 $[9,10]$. In this work, the calculations are performed using the RHB model with separable pairing force and spherical symmetry is assumed. It is seen that all interactions under consideration provide a reasonable description of the binding energies and produce similar isotopic dependencies. Compared to the DD-PC1 and DD-ME2, the newly parametrized DD-PCX interaction seems to be more successful in the predictions of the experimental binding energies of spherical nuclei. Since 

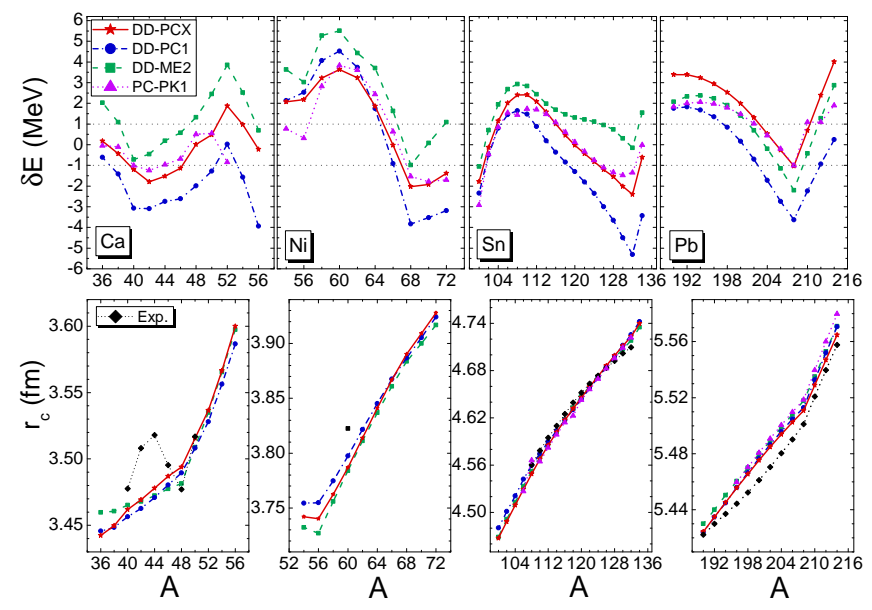

FIG. 1. Upper panel: the difference between the experimental [39] and calculated binding energies for $\mathrm{Ca}, \mathrm{Ni}, \mathrm{Sn}$ and $\mathrm{Pb}$ isotopes. Lower panel: the calculated charge radii in comparison with the experimental values [40]. The calculations are performed using the DD-PCX, DD-PC1 [7], and DD-ME2 [8] interactions. Available data for the PC-PK1 interaction from Refs. [9, 10] are also shown.

the pairing parameters $\left(G_{n}, G_{p}\right)$ are also included in the fitting protocol, the success of the DD-PCX can also be related to the better reproduction of pairing properties of nuclei as discussed below. In comparison to the experimental data [40], the charge radii are also accurately reproduced, with a few exceptions for $\mathrm{Ca}$ isotopes and ${ }^{60} \mathrm{Ni}$. In order to assess a more general overview about the performance of the DD-PCX interaction, we calculate the root mean square error $(\Delta)$ and root mean square relative error $(\delta)$ in percentage for binding energies, charge radii, and mean pairing gaps for a set of nuclei (see supplementary material for the list of nuclei), and the results are given in Table III. It is seen that the resulting binding energies and mean gap values are better reproduced using the DD-PCX interaction, while the deviations for the charge radii are similar in all cases. An extended list with the calculated binding energies and charge radii for the selected nuclei using the same interactions is also given in the supplemental material with the corresponding experimental data.

The predictive power of the DD-PCX interaction is also tested on the nuclear excitations of nuclei. Table IV shows the constrained ISGMR energies for ${ }^{90} \mathrm{Zr},{ }^{120} \mathrm{Sn}$, ${ }^{208} \mathrm{~Pb}$ calculated using various relativistic interactions, and compared to the experimental results [34, 35, 4143]. In this part, we should mention that the DD-ME2 calculations are performed using the finite range Gogny interaction D1S in the particle-particle channel for openshell nuclei [44], while separable pairing is used with the DD-PCX and DD-PC1 interactions. Among the relativistic interactions, the DD-ME2 (DD-PC1) interaction
TABLE III. The root mean square error $(\Delta)$ and root mean square relative error $(\delta)$ in percentage for the binding energies (B.E.) $(\mathrm{MeV})$, charge radii $\left(r_{c}\right)(\mathrm{fm})$ and mean gaps $(\mathrm{MeV})$ for a set of spherical nuclei, using the relativistic DD-PCX, DDPC1 and DD-ME2 interactions. The numbers of the nuclei considered in the calculations are given in parentheses.

\begin{tabular}{ccccc}
\hline \hline Interaction & & $\begin{array}{c}\text { B.E. } \\
(65)\end{array}$ & $\begin{array}{c}r_{c} \\
(46)\end{array}$ & $\begin{array}{c}\text { Mean Gap } \\
(56)\end{array}$ \\
\hline \multirow{2}{*}{ DD-PCX } & $\Delta$ & $1.38 \mathrm{MeV}$ & $0.016 \mathrm{fm}$ & $0.18 \mathrm{MeV}$ \\
& $\delta$ & $0.21 \%$ & $0.47 \%$ & $15.40 \%$ \\
\hline \multirow{2}{*}{ DD-PC1 } & $\Delta$ & $3.05 \mathrm{MeV}$ & $0.017 \mathrm{fm}$ & $0.29 \mathrm{MeV}$ \\
& $\delta$ & $0.48 \%$ & $0.49 \%$ & $21.73 \%$ \\
\hline \multirow{2}{*}{ DD-ME2 } & $\Delta$ & $2.08 \mathrm{MeV}$ & $0.016 \mathrm{fm}$ & $0.35 \mathrm{MeV}$ \\
& $\delta$ & $0.27 \%$ & $0.44 \%$ & $26.13 \%$ \\
\hline
\end{tabular}

predicts the lowest (highest) values for the constrained ISGMR energies. Considering the point coupling interactions, the table clearly demonstrates the relevance of including the constraint on the ISGMR excitation energy for ${ }^{208} \mathrm{~Pb}$ in order to provide a reasonable description of the ISGMR energies in all nuclei under consideration. Using the DD-PCX interaction, the calculated values for ${ }^{90} \mathrm{Zr}$ and ${ }^{120} \mathrm{Sn}$ are slightly above the experimental values and further fine-tuning of the interaction may be achieved by considering the ISGMR energies of additional nuclei and using smaller adopted error within the $\chi^{2}$ minimization of the interaction. However, the softness of the Sn nuclei still represents an open question, and their inclusion in constraining the interaction may cause difficulties.

TABLE IV. The constrained ISGMR energies (in $\mathrm{MeV}$ ) for ${ }^{90} \mathrm{Zr},{ }^{120} \mathrm{Sn}$ and ${ }^{208} \mathrm{~Pb}$ in comparison with the experimental data.

\begin{tabular}{ccccc}
\hline & Exp. & DD-PCX & DD-PC1 & DD-ME2 \\
\hline${ }^{90} \mathrm{Zr}$ & $17.58_{-0.04}^{+0.06}[41]$ & $18.00 \pm 0.10$ & 18.83 & 17.80 \\
& $17.66 \pm 0.07[42]$ & & & \\
${ }^{120} \mathrm{Sn}$ & $15.5 \pm 0.1[43]$ & $16.18 \pm 0.09$ & 16.92 & 16.07 \\
${ }^{208} \mathrm{~Pb}$ & $13.5 \pm 0.1[34]$ & $13.66 \pm 0.08$ & 14.22 & 13.49 \\
& $14.18 \pm 0.11[35]$ & & & \\
\hline
\end{tabular}

Due to the empirical constraint imposed on the dipole polarizability for ${ }^{208} \mathrm{~Pb}$, it is expected that the isovector channel of the DD-PCX interaction is improved in comparison to other effective interactions, as illustrated in a few following examples. Figure 2 shows the dipole polarizabilities for ${ }^{48} \mathrm{Ca},{ }^{68} \mathrm{Ni}$ and ${ }^{208} \mathrm{~Pb}$ (left panel) and Sn isotopic chain (right panel). The calculations are performed using the DD-PCX, DD-PC1 and DD-ME2 interactions, and the available experimental data are also provided [18, 20-23]. As shown in Fig. 2, only the DD-PCX 


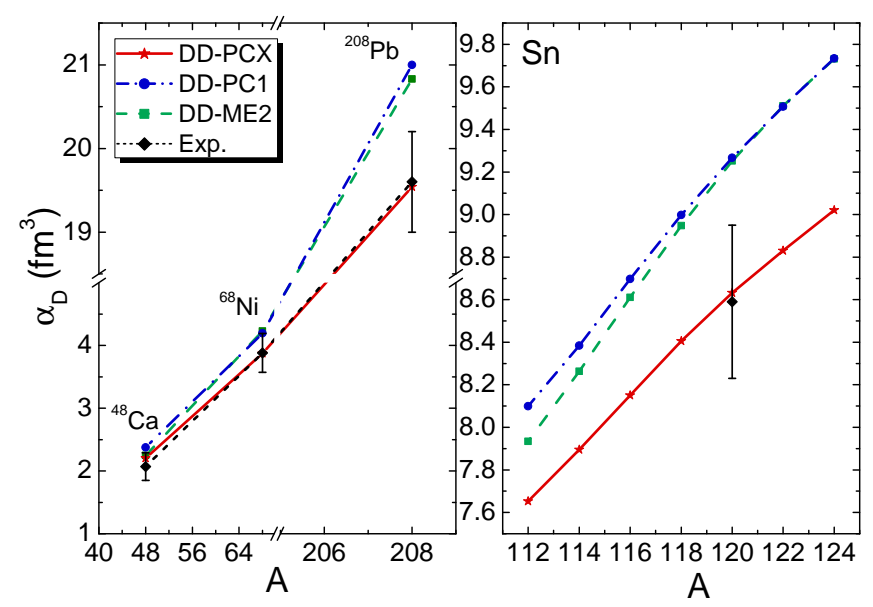

FIG. 2. The dipole polarizabilities for ${ }^{48} \mathrm{Ca},{ }^{68} \mathrm{Ni}$ and ${ }^{208} \mathrm{~Pb}$ (left panel) and tin isotopic chain (right panel). The calculations are performed using RHB+(Q)RPA with DD-PCX, DD-PC1 and DD-ME2 interactions. The experimental data are taken from Refs. [18, 20-23].

interaction systematically reproduces the experimental results on $\alpha_{D}$ for all nuclei under consideration, while the DD-ME2 and DD-PC1 interactions mainly overestimate the measured values. Although in constraining the functional we only use the dipole polarizability for ${ }^{208} \mathrm{~Pb}$, the DD-PCX interaction is also successful in the prediction of the dipole polarizabilities for other nuclei, as expected due to the strong correlation between the dipole polarizabilities in different nuclei [18].

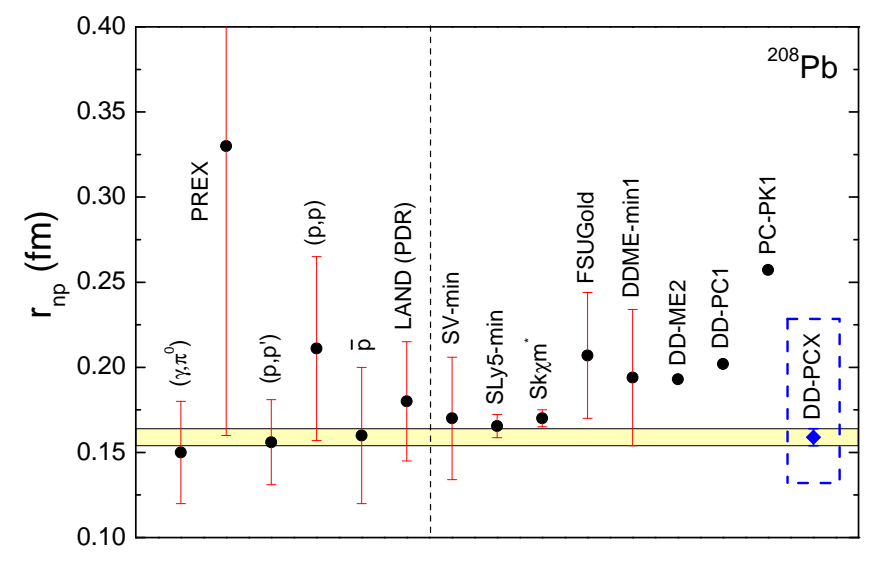

FIG. 3. The neutron skin thickness for ${ }^{208} \mathrm{~Pb}$ predicted by different experiments and nuclear energy density functionals. The experimental data are taken from $\left(\gamma, \pi^{0}\right)$ [47], PREX [17], $\left(p, p^{\prime}\right)$ [20], $(p, p)$ [48], $\bar{p}$ [49], LAND(PDR) [50]. The calculations with the non-relativistic interactions: SV-min [4], SLy5min [32], Sk $\chi m^{*}$ [46], and relativistic interactions: FSUGold [45], DDME-min [32], DD-ME2 [8], DD-PC1 [7], PC-PK1 [9].
Another important isovector property to be considered is the neutron skin thickness $r_{n p}$. In Fig. 3, the neutron skin thickness for ${ }^{208} \mathrm{~Pb}$ is shown for a set of relativistic [7-9, 32, 45] and non-relativistic calculations [4, 32, 46] along with the experimental data from Refs. [17, 20, 4750]. Using the DD-PCX interaction, the neutron skin thickness for ${ }^{208} \mathrm{~Pb}$ is predicted as $r_{n p}=0.159 \pm 0.005 \mathrm{fm}$, and the (yellow) band denotes the calculated statistical uncertainty. Considering the experimental results, it is clear that there are discrepancies in the predictions for $r_{n p}$ values in ${ }^{208} \mathrm{~Pb}$. The non-relativistic functionals predict lower values for the neutron skin thickness compared to the relativistic ones, with the exception of the new effective interaction DD-PCX. Among the relativistic interactions, the DD-PCX prediction provides the lowest neutron skin thickness for ${ }^{208} \mathrm{~Pb}$, which is also in a good agreement with the majority of the experimental data. The neutron-skin thicknesses of neutron-rich ${ }^{48} \mathrm{Ca}$ and ${ }^{132} \mathrm{Sn}$ nuclei are also calculated as $0.172 \pm 0.003 \mathrm{fm}$ and $0.218 \pm 0.005 \mathrm{fm}$, respectively. We find that the calculated neutron skin thicknesses are also in a good agreement with the "model-averaged" results in Ref. [51]. The results demonstrate that the DD-PCX interaction can also be used to make reliable predictions for the neutron skin thicknesses of other nuclei.

In conclusion, we have established a unified theoretical framework to constrain the relativistic nuclear EDF, based on the RHB plus (Q)RPA, supplemented with the co-variance analysis, using not only the nuclear ground state properties, but also relevant properties on collective nuclear excitations. The relativistic point-coupling interaction DD-PCX introduced in this work, represents the first effective interaction that is constrained using the binding energies, charge radii and pairing gaps, together with a direct implementation of the ISGMR energy and dipole polarizability in the $\chi^{2}$ minimization. In comparison to the previous studies, where the functionals have the properties of the nuclear symmetry energy either unconstrained, constrained by the pseudo-observables on nuclear matter that are often arbitrary, or validated by the data after the parameters have been determined, the present study implements directly genuine observables on collective excitations to optimize the effective nuclear interaction. The success of the DD-PCX interaction in the predictions of the dipole polarizabilities and neutron skin thicknesses in other nuclei not used in optimizing the model parameters validates the isovector channel of the functional and the respective symmetry energy properties. The present analysis clearly demonstrates the relevance of accurate measurements of the nuclear collective phenomena, as well as the necessity to resolve the ambiguities in the existing data from different experiments, both in the isoscalar (e.g., the ISGMR energy in ${ }^{208} \mathrm{~Pb}[34,35]$ and isovector sectors for constraining modern nuclear energy density functionals.

We thank T. Nikšić for stimulating discussions while 
preparing this paper. This work is supported by the Croatian Science Foundation under the project Structure and Dynamics of Exotic Femtosystems (IP-201409-9159) and by the QuantiXLie Centre of Excellence, a project co financed by the Croatian Government and European Union through the European Regional Development Fund, the Competitiveness and Cohesion Operational Programme (KK.01.1.1.01). E. Y. also acknowledges financial support from the Scientific and Technological Research Council of Turkey (TÜBITTAK) BIDEB2219 Postdoctoral Research program.

* eyuksel@yildiz.edu.tr

$\dagger$ Current affiliation: Ericsson Nikola Tesla d.d., Krapinska 45, 10000 Zagreb, Croatia

‡ npaar@phy.hr

[1] T. Nikšić, D. Vretenar, P. Ring, Prog. Part. Nucl. Phys. 66, 519 (2011).

[2] N. Paar, D. Vretenar, E. Khan, and G. Colò, Rep. Prog. Phys. 70, 691 (2007).

[3] X. Roca-Maza and N. Paar, Prog. Part. Nucl. Phys. 101, 96-176 (2018).

[4] P. Klüpfel, P. -G. Reinhard, T. J. Bürvenich, and J. A. Maruhn, Phys. Rev. C 79, 034310 (2009).

[5] S. Goriely, S. Hilaire, M. Girod, and S. Pèru, Phys. Rev. Lett. 102, 242501 (2009).

[6] M. Kortelainen, T. Lesinski, J. Morè, W. Nazarewicz, J. Sarich, N. Schunck, M. V. Stoitsov, and S. Wild, Phys. Rev. C 82, 024313 (2010).

[7] T. Nikšić, D. Vretenar, and P. Ring, Phys. Rev. C 78, 034318 (2008).

[8] G. A. Lalazissis, T. Nikšić, D. Vretenar, and P. Ring, Phys. Rev. C 71, 024312 (2005).

[9] P. W. Zhao, Z. P. Li, J. M. Yao, and J. Meng, Phys. Rev. C 82, 054319 (2010).

[10] P. W. Zhao, private communications (2015).

[11] W. -C. Chen and J. Piekarewicz, Phys. Rev. C 90, 044305 (2014).

[12] J. Dobaczewski, J. Phys. G 43 04LT01 (2016).

[13] J. Bonnard, M. Grasso, and D. Lacroix, Phys. Rev. C 98, 034319 (2018).

[14] S. Shen, H. Liang, J. Meng, P. Ring, and S. Zhang, Phys. Lett. B 778, 344 (2018).

[15] M. Baldo and G. F. Burgio, Prog. Part. Nucl. Phys. 91, 203 (2016).

[16] J. Piekarewicz, Eur. Phys. J. A 50, 25 (2014).

[17] S. Abrahamyan et al., Phys. Rev. Lett. 108, 112502 (2012).

[18] X. Roca-Maza, X. Viñas, M. Centelles, B. K. Agrawal, G. Colò, N. Paar, J. Piekarewicz, and D. Vretenar, Phys. Rev. C 92, 064304 (2015).
[19] P. -G. Reinhard and W. Nazarewicz, Phys. Rev. C 81, 051303 (2010).

[20] A. Tamii et al., Phys. Rev. Lett. 107, 062502 (2011).

[21] J. Birkhan et al., Phys. Rev. Lett. 118, 252501 (2017).

[22] T. Hashimoto et al., Phys. Rev. C 92, 031305 (2015).

[23] D. M. Rossi et al., Phys. Rev. Lett. 111, 242503 (2013).

[24] U. Garg and G. Colò, Prog. Part. Nucl. Phys. 101, 55 (2018).

[25] W. Kohn and L.J. Sham, Phys. Rev. 140, A1133 (1965).

[26] W. Kohn, Rev. Mod. Phys. 71, 1253 (1999).

[27] T. Nikšić, N. Paar, D. Vretenar, and P. Ring, Comput. Phys. Commun. 185, 1808 (2014).

[28] D. R. Entem, R. Machleidt, Phys. Rev. C 68, 041001(R) (2003)

[29] Y. Tian, Z. Y. Ma, and P. Ring, Phys. Rev. C 80, 024313 (2009).

[30] D. Vretenar, A. V. Afanasjev, G. A. Lalazissis, and P. Ring, Phys. Rep. 409, 101 (2005).

[31] J. Meng, H. Toki, S. G. Zhou, S. Q. Zhang, W. H. Long, L. S. Geng, Prog. Part. Nucl. Phys. 57, 470-563 (2006).

[32] X. Roca-Maza, N. Paar, and G. Colò, J. Phys. G 42, 034033 (2015).

[33] M. Bender, K. Rutz, P. -G. Reinhard, and J. A. Maruhn, Eur. Phys. J. A 8, 59 (2000).

[34] D. Patel et al., Phys. Lett. B 726, 178 (2013).

[35] D. H. Youngblood, H. L. Clark, and Y. -W. Lui, Phys. Rev. Lett. 82, 691 (1999).

[36] D. Patel et al., Phys. Lett. B 735, 387 (2014).

[37] M. Uchida et al., Phys. Rev. C 69051301 (2004).

[38] D. H. Youngblood, Y. -W. Lui, H. L. Clark, B. John, Y. Tokimoto, X. Chen, Phys. Rev. C 69, 034315 (2004).

[39] M. Wang, G. Audi, F. G. Kondev, W. J. Huang, S. Naimi, and X. Xu, Chin. Phys. C 41, 030003 (2017).

[40] I. Angeli and K. P. Marinova, At. Data Nucl. Data Tables 99, 69 (2013).

[41] Krishichayan, Y. -W. Lui, J. Button, D. H. Youngblood, G. Bonasera, S. Shlomo, Phys. Rev. C 92, 044323 (2015).

[42] Y. K. Gupta et al., Phys. Lett. B 760, 482 (2016).

[43] T. Li et al., Phys. Rev. C 81, 034309 (2010).

[44] N. Paar, T. Nikšić, D. Vretenar and P. Ring, Phys. Rev. C 67, 034312 (2003).

[45] B. G. Todd-Rutel and J. Piekarewicz, Phys. Rev. Lett. 95, 122501 (2005).

[46] Z. Zhang, Y. Lim, J. W. Holt, C. -M Ko, Phys. Lett. B 777, 73 (2018).

[47] C. M. Tarbert et al. (Crystal Ball at MAMI and A2 Collaboration), Phys. Rev. Lett. 112, 242502 (2014).

[48] J. Zenihiro et al., Phys. Rev. C 82, 044611 (2010); J. Zenihiro; PhD Thesis, Kyoto University, 2011.

[49] B. Kłos et al., Phys. Rev. C 76, 014311 (2007).

[50] A. Klimkiewicz et al., Phys. Rev. C 76, 051603 (2007).

[51] J. Piekarewicz, B. K. Agrawal, G. Colò, W. Nazarewicz, N. Paar, P. -G. Reinhard, X. Roca-Maza, and D. Vretenar, Phys. Rev. C 85, 041302 (2012). 